\title{
Predicting Target Vessel Location for Improved Planning of Robot-Assisted CABG Procedures
}

\author{
Daniel S. Cho ${ }^{1,2}$, Cristian A. Linte ${ }^{1,2}$, Elvis Chen ${ }^{1}$, Chris Wedlake ${ }^{1}$, \\ John Moore ${ }^{1}$, John Barron ${ }^{4}$, Rajni Patel ${ }^{3}$, and Terry M. Peters ${ }^{1,2,3}$ \\ ${ }^{1}$ Imaging Research Laboratories, Robarts Research Institute \\ ${ }^{2}$ Biomedical Engineering Graduate Program, University of Western Ontario \\ ${ }^{3}$ Canadian Surgical Technologies and Advanced Robotics \\ ${ }^{4}$ Department of Computer Science, University of Western Ontario, \\ London, Ontario, Canada
}

\begin{abstract}
Prior to performing a robot-assisted coronary artery bypass grafting procedure, a pre-operative computed tomography scan is used to assess patient candidacy and to identify the location of the target vessel. The surgeon then determines the optimal port locations to ensure proper reach to the target with the robotic instruments, while assuming that the heart does not undergo any significant changes between the preand intra-operative stages. However, the peri-operative workflow itself leads to changes in heart position and consequently the intra-operative target vessel location. As such, the pre-operative plan must be adequately updated to adjust the target vessel location to better suit the intraoperative condition. Here we propose a technique to predict the position of the peri-operative target vessel location with $\sim 3.5 \mathrm{~mm}$ RMS accuracy. We believe this technique will potentially reduce the rate of conversion of robot-assisted procedures to traditional open-chest surgery due to poor planning.
\end{abstract}

\section{Introduction}

The quest for alternate approaches to conventional cardiac therapy has increased the number of minimally invasive procedures being performed, leading to less trauma, shorter hospital stays, faster return to normal activities, and improved cosmesis [1|2 34 . Robot-assisted (RA) surgery represents a paradigm shift in the delivery of health care for both the patient and the surgeon [5] and it has been adopted as standard of care at many institutions worldwide [5]; one of the popular cardiac interventions performed under robot-assistance is the coronary artery bypass grafting (CABG) procedure.

In current clinical practice, a pre-operative computed tomography (CT) scan of the patient is used to assess his/her candidacy for undergoing a RA-CABG procedure. Based on the pre-operative scan, the surgeon identifies the location of the surgical target — the left anterior descending (LAD) coronary artery, examines whether there is sufficient workspace inside the chest wall for the robot arms, and ultimately estimates the optimal locations of the port incisions to

T. Jiang et al. (Eds.): MICCAI 2010, Part III, LNCS 6363, pp. 205-212, 2010.

(C) Springer-Verlag Berlin Heidelberg 2010 
ensure proper reach of the surgical targets with the robotic instruments. However, it is not unusual that after setting up the patient for the robot-assisted procedure, the surgeons encounter difficulties due to the inability in reaching the target, robot arm collisions or reduced dexterity [6]. In fact, 20-30\% of the RA-CABG interventions require conversion to traditional open-chest surgery [7], mainly due to the migration of the heart during the peri-operative workflow, not accounted for in the pre-operative plan.
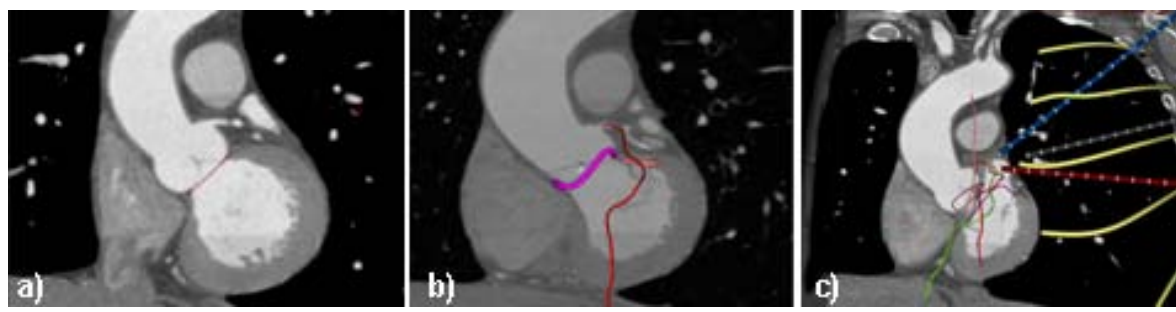

Fig. 1. Pre-operative planning stage showing patient's cardiac CT scan (a), the coronary vessel displayed relative to the valve annuli (b) and the port placement to ensure proper reach of the target vessel with the robotic instruments (c) Note: the yellow lines represent intercostal spaces.

It is believed that the workflow stages - lung collapse and $\mathrm{CO}_{2}$ chest insufflation - will induce an overall shift of the heart inside the thoracic cavity, which may invalidate the surgical plan based solely on the pre-operative data. Therefore, is critical to estimate the global heart displacement during the typical peri-operative workflow and improve the pre-operative plan by estimating the intra-operative location of the target vessel. However, the global migration of the heart has never been measured, nor has the change in location of the LAD between the pre- and intra-operative stages. The contribution of this paper is twofold. First, we provide an estimate of the heart migration measured from clinical patient data by registering the pre-operative cardiac CT (containing the LAD location identified from the CT dataset - Fig. 1) to images acquired peri-operatively using tracked US. Secondly, to overcome the clinical limitation arising due to the invisibility of the LAD in the US images, we conducted an in vitro study where we simulated the clinically observed heart shift and demonstrated the feasibility of our technique towards predicting the LAD location.

While no known accuracy constraints have been reported for this specific application, our collaborating cardiac surgeons have recommended that a target prediction accuracy on the order of one intercostal space $(\sim 10-15 \mathrm{~mm}$, depending on patient size) is desired. From a clinical feasibility perspective, this constraint is valid: as long as the intra-operative LAD location is correctly predicted to within one intercostal space from its actual location, it can be reached by positioning the trocar on either side of the adjacent rib. 


\section{Materials and Methods}

\subsection{Clinical Procedure Workflow}

In a typical RA-CABG procedure, the patient is first imaged pre-operatively $\left(\right.$ Stage $\left._{0}\right)$ in the same position as during the intervention. Peri-operatively, following intubation and anesthesia delivery ( Stage $_{1}$ ), the left lung is collapsed $\left(\right.$ Stage $\left._{2}\right)$, and the chest is insufflated $\left(\right.$ Stage $\left._{3}\right)$ to provide sufficient work space.

Image Acquisition. We employed real-time 2D trans-esophageal echocardiography (TEE) to monitor the heart during the interventional workflow. The images were acquired using a spatially tracked TEE probe modified by embedding a 6 DOF Aurora magnetic sensor coil (Northern Digital Inc., Waterloo, Canada) inside the encasing of the transducer 88. Images were collected from three different views in order to capture all of the necessary cardiac features. For our work, mid-esophageal-4-chamber view images were captured at $20^{\circ}$ increments from $0^{\circ}$ to $180^{\circ}$ for the mitral valve annulus (MVA) and the left ventricular apex (LVAp). Five long-axis view images with $10^{\circ}$ increments and one short-axis view of the aorta at $30^{\circ}$ were also acquired to visualize the aortic valve annulus (AVA) and the left coronary ostium (LCO). US image data were acquired by an expert anesthetist and repeated three times at each workflow stage.
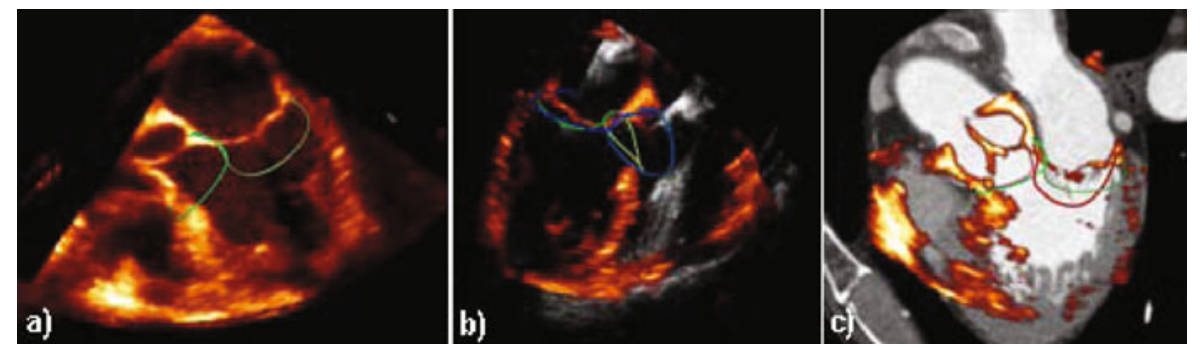

Fig. 2. a) Patient heart instance at Stage ${ }_{1}$ acquired using tracked US and showing the valve annuli; b) Stage ${ }_{1}$ (orange) and Stage $_{2}$ (gray) instances of the heart showing relative heart displacement and corresponding segmented valvular structures; c) Initial peri-operative US instance (Stage ${ }_{1}$ ) registered to the pre-operative dataset and displayed within the CT coordinate space.

Estimating Global Heart Displacement. The peri-operatively acquired instances were then transferred into the CT coordinate system by aligning homologous features corresponding to the first peri-operative (Stage ${ }_{1}$ US) and the pre-operative (Stage CT$_{0}$ ) datasets (Fig. 2). These two stages are physiologically equivalent given the same patient position and dual-lung ventilation, and hence minimal anatomical variations are expected. As a result, the peri-operative displacements can be estimated with respect to the principal body axes. 


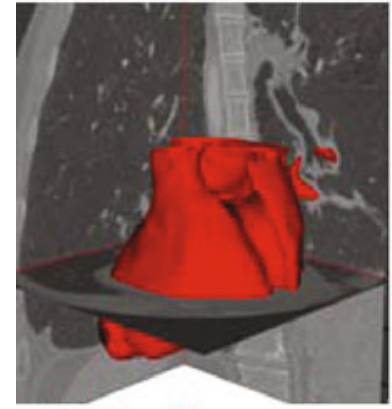

St 1: Dual-lung vent.

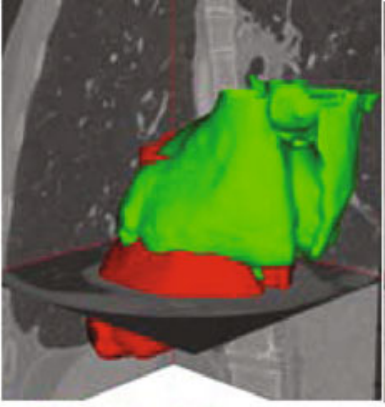

St 2: Single-lung vent.

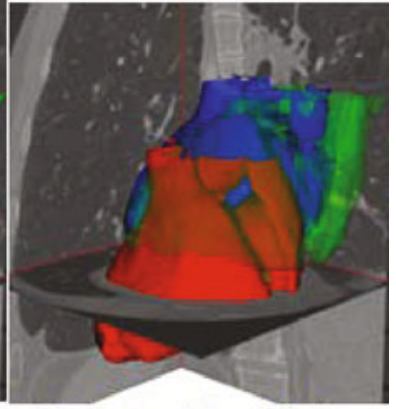

St 3: Chest insufflation

Fig. 3. Visual representation showing an automatically segmented epicardial model of a patient's heart animated using the sequential peri-operative transforms based on the valvular structures. Note: Stage ${ }_{1}$ is shown in red, $\mathrm{Stage}_{2}$ in green and $\mathrm{Stage}_{3}$ in blue.

We have shown the migration patterns in four patients undergoing RA-CABG procedure [9]. Our clinical data have suggested that the heart undergoes considerable displacement during the workflow, which should not be ignored during the planning process. As an example, we show the change in position of the epicardial surface of one patient's heart segmented from the CT dataset and animated using the sequential peri-operative transforms (Fig. 3). Moreover, in spite of the observed displacements, the morphology of the identified features remains relatively consistent throughout the workflow 9, suggesting that no significant non-rigid deformations are occurring. Based on these clinical observations, we next conducted an in vitro validation study to assess the accuracy with which a newly-developed technique involving a rigid-body feature-based registration can predict the peri-operative location of the LAD vessel.

\subsection{Predicting the LAD Location: Feature-Based Registration}

Since the LAD can only be clearly seen in the pre-operative CT image and not in the peri-operative US images, its peri-/intra-operative location has to be deduced based on the rest of the data available peri-operatively. Therefore, we chose to predict its location via a registration algorithm that involves four features - LCO, MVA, AVA and LVAp - easily identifiable in both modalities and sufficiently close to the target vessel to provide improved accuracy in the region of interest. The LAD begins at the left coronary ostium and usually runs toward the apex, while the mitral and aortic valves are located on either side of the starting point of the LAD. A rigid-body registration driven by the above features was then applied to map the pre-operative dataset to the peri-operative datasets, therefore predicting the LAD location at each subsequent stage. The Euclidean distance between each set of homologous features was initially computed, followed by the optimization of the cost function shown in Eq. 1 performed using the downhill simplex method [10].

$$
\text { cost }=\alpha \operatorname{dist}_{L C O}+\beta \operatorname{dist}_{L V A p}+\gamma\left(\operatorname{dist}_{M V A}+\operatorname{dist}_{A V A}\right)
$$


Since distances between the LCO and the LVAp were selected as the main constraints for the registration, the optimal values for the weighting factor parameters were emphirically determined as follows: $\alpha=1.0, \beta=1.0$, and $\gamma=0.6$, yielding an optimal alignment.

\subsection{In vitro Experimental Validation}

Experimental Apparatus: Considering that the LAD cannot be identified peri-operatively using US imaging, we conducted an in vitro validation study to assess the accuracy with which our technique can predict the LAD location. The experimental apparatus was set up in a configuration similar to that typically found in the OR: we simulated the migration patterns of the heart induced during RA-CABG procedures by altering the position and orientation of a heart phantom (The Chamberlain Group, Great Barrington, USA).

Sixteen CT-visible fiducials were attached to the surface of the phantom: ten were used to assist with the CT-to-phantom registration and the remaining six were used to "define" the path of the LAD vessel. The position of the heart phantom was tracked throughout the study using a 6-DOF NDI Aurora magnetic sensor rigidly attached onto the phantom. Two different modalities (CT and US) were employed for image acquisition: a pre-operative CT scan was acquired and a virtual surface model was constructed using automatic segmentation tools; peri-operative images at each workflow stage were acquired using a magneticallytracked TEE probe similar to the one used in the OR (Fig. 4). The LAD vessel was initially identified from the CT image and its peri-operative location was predicted based on its pre-operative location using the proposed registration.
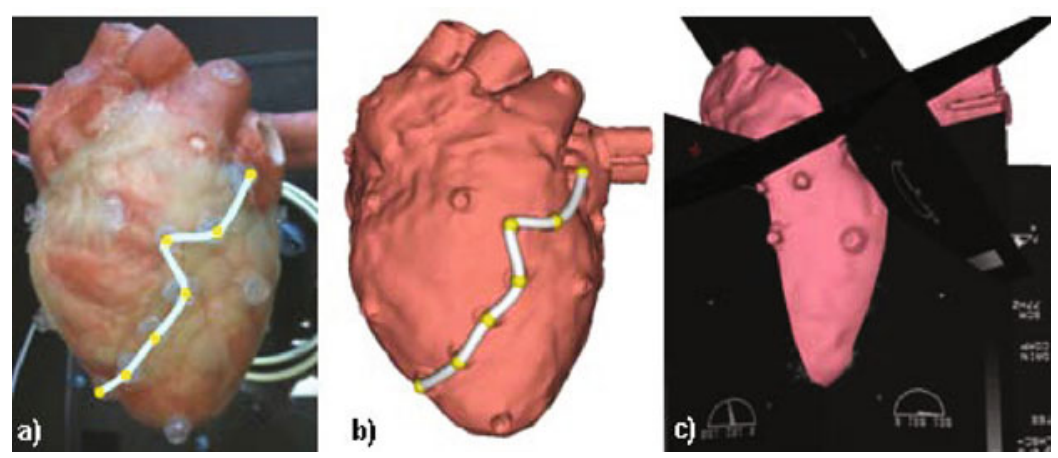

Fig. 4. a) Image and (b) virtual surface model of the heart phantom showing the LAD path; c) Peri-operative US image acquisition protocol showing imaging of the apex and coronary ostia using incrementally tracked 2D US images.

Intra-operative Image Acquisition: Since TEE is the standard of care for monitoring during cardiac procedures, in this study we collected the required tracked US images following the clinical workflow. The position of the heart 
was altered twice, to mimic the actual intervention (i.e. lung collapse and chest insufflation). In each position, the image acquisition was repeated three times and the entire protocol was also repeated three times to minimize human errors. The four features were then extracted from the images using a custom-developed segmentation tool. All features were defined in the same 3D coordinate space; the mitral and aortic valves were represented as "rings", while the ostium and the apex were represented as points.

Assessing Intra-operative Target Vessel Location: The six fiducials positioned along the LAD path were used to assess the target registration error (TRE) between the predicted LAD fiducial locations and their gold-standard locations, at each stage in the workflow. The gold-standard LAD fiducial locations were determined by recording the LAD fiducial locations at each stage using a magnetically tracked pointer and confirmed using the point-based registration transform corresponding to each peri-operative stage. The predicted LAD location was identified by mapping the pre-operative LAD fiducials using the feature-based registration transform described in section $\mathbf{2 . 2}$.

\section{Evaluation and Results}

We simulated three different RA-CABG-related workflows by altering the position and orientation of the heart phantom at three different stages. For each of the nine poses, we acquired three sets of tracked US images, defined the features of interest, and used the proposed registration algorithm to predict the location of the LAD vessel. Table 1 summarizes the TRE between the actual locations of the LAD target fiducials (the ground truth) and their predicted locations from the registration.

Table 1. LAD TRE: Mean \pm SD and RMS (mm)

\begin{tabular}{|c|c|c|c|c|c|c|c|c|}
\hline \multirow{2}{*}{\begin{tabular}{|l|} 
LAD \\
Point
\end{tabular}} & \multicolumn{2}{|c|}{ Stage $_{0}$ to Stage $_{1}$} & \multicolumn{2}{|c|}{ Stage $_{0}$ to Stage $_{2}$} & \multirow{4}{*}{\multicolumn{4}{|c|}{$\begin{array}{l}\text { Table 2. RMS Feature Localiza- } \\
\text { tion Error }(\mathrm{mm})\end{array}$}} \\
\hline & Mean \pm SD & RMS & Mean \pm SD & RMS & & & & \\
\hline 1 & $3.1 \pm 0.9$ & \begin{tabular}{|l|}
3.2 \\
\end{tabular} & $2.9 \pm 1.7$ & \begin{tabular}{|l|}
3.4 \\
\end{tabular} & & & & \\
\hline 2 & $2.9 \pm 1.3$ & 3.2 & $3.4 \pm 1.6$ & 3.8 & & & & \\
\hline 3 & $3.4 \pm 1.4$ & 3.7 & $3.3 \pm 1.6$ & 3.7 & Feature & Stage $_{0}$ & Stage $_{1}$ & Stage $_{2}$ \\
\hline 4 & $3.9 \pm 1.9$ & 4.3 & $3.8 \pm 1.3$ & 4.0 & \begin{tabular}{|l|} 
LVAp \\
\end{tabular} & \begin{tabular}{|l|}
2.3 \\
\end{tabular} & \begin{tabular}{|l|l|}
4.7 \\
\end{tabular} & \begin{tabular}{|l|} 
\\
4.0
\end{tabular} \\
\hline 5 & $4.4 \pm 2.3$ & 5.0 & $3.9 \pm 1.7$ & 4.3 & AVA & 1.4 & 1.3 & 1.2 \\
\hline 6 & $4.9 \pm 2.6$ & 5.5 & $4.5 \pm 2.1$ & 5.0 & MVA & 0.6 & 1.9 & 1.5 \\
\hline Overall & $3.7 \pm 1.9$ & 4.2 & $3.6 \pm 1.7$ & 4.0 & $\mathrm{CO}$ & 1.1 & 4.0 & 2.1 \\
\hline
\end{tabular}

For a visual interpretation of the LAD TRE, Fig. 5 shows the virtual model of the heart phantom along with the gold-standard and predicted LAD paths at both Stage $_{1}$ and Stage $_{2}$ in the peri-operative workflow, showing clinicallyadequate alignment, well under the $10-15 \mathrm{~mm}$ intercostal space constraint. 

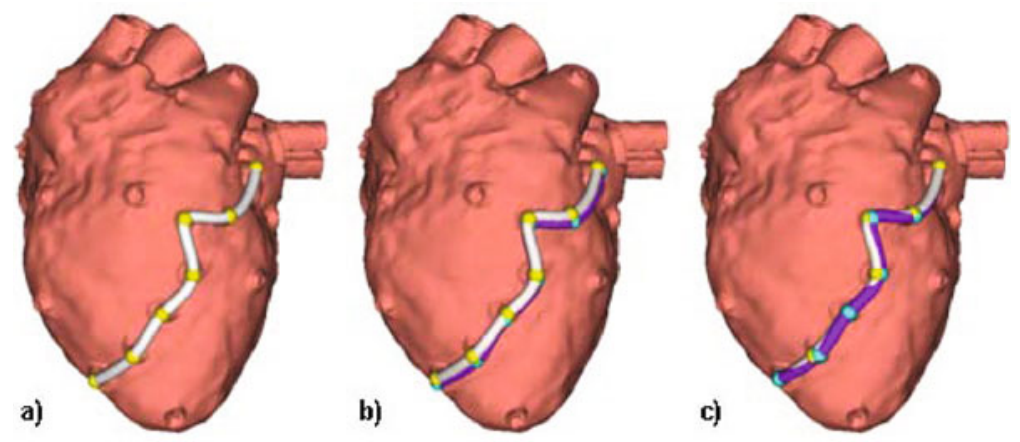

Fig. 5. a) Pre-operative heart phantom model at Stage 0 showing the LAD vessel; Visual display of the LAD TRE at Stage $_{1}$ (b) and Stage 2 (c), showing the gold-standard LAD (seashell white) obtained using the point-based registration transform and the predicted LAD (purple) determined using the proposed feature-based registration transform.

\section{Discussion}

This work constitutes the first steps towards optimizing pre-operative planning for RA-CABG procedures. Motivated by a recent clinical study that revealed substantial migration of the heart during the peri-operative procedure workflow, our goal is to predict the intra-operative location of the target vessel, to provide the surgeon with an optimized surgical plan that better reflects the intra-operative stage.

As a bridge to the in vivo validation, considering the limitations arising due to poor visualization and identification of the LAD coronary vessel in clinical US images, the in vitro phantom study was performed to assess the accuracy with which the proposed feature-based registration technique can predict the location of a target vessel. Our results have shown a root-mean-squared (RMS) TRE on the order of $\sim 3.5 \mathrm{~mm}$ across the twenty-seven peri-operative poses simulated in our study. Moreover, considering that the target vessel location is predicted using a feature-based registration algorithm, we next assessed the error associated with the feature localization. Table 2 includes a summary of the RMS localization error associated with the identification of each of the four features used to drive the registration: LCO, LVAp, MVA and AVA. As observed, the point-based localization of the LVAp and the LCO was consistently challenging, mainly due to the 2D nature of the US images used to identify a 3D structure.

These feature localization errors in fact explain the increasing TRE at the LAD fiducials closer to the apical region at both peri-operative stages. Considering that the anastomosis target site is typically located along the LAD path two thirds of the way from the ostia towards the apex, we have implemented a more robust apex localization approach from the US data which led to improved target registration error near the inferior end of the LAD. The new algorithm uses the apical region as a registration constraint as opposed to a single point, and include a robust estimator to reduce the TRE, as suggested by Ma et al. [11. 
Nevertheless, in spite of these slight inaccuracies, our results are well within the 10-15 mm clinically-imposed constraint dictated by a typical intercostal space, allowing sufficient tolerance (over $10 \mathrm{~mm}$ ) in the event that these errors amplify when using clinical data.

\section{Conclusions}

Driven by the clinical motivation to improve the pre-operative planning of RACABG procedures, here we have proposed and evaluated a technique used to predict the intra-operative target vessel location. Our technique was validated in an in vitro study simulating the clinically-observed RA-CABG procedure workflow and yielded $3.5 \mathrm{~mm}$ RMS accuracy in predicting the peri-operative LAD location. These results agree with the clinical constraints imposed by this application and provided an equally successful in vivo evaluation in our upcoming animal studies, currently under research ethics approval, employing DynaCT (Siemens, Erlangen, Germany) for intra-operative validation, we believe this technique has the potential to significantly improve the current pre-operative planning of RA-CABG procedures, and consequently lead to reduced rates of conversion to traditional open-chest surgery.

\section{References}

1. Modi, P., Hassan, A., Chitwood, W.R.J.: Minimally invasive mitral valve surgery: a systematic review and meta-analysis. Eur. J. Cardiothorac. Surg. 34, 943-952 (2008)

2. Doty, D.B., Flores, J.H., Doty, J.R.: Cardiac valve operations using a partial sternotomy technique. J. Card. Surg. 15, 35-42 (2000)

3. Vassiliades, T.A., Block, P.C., Cohn, L.H., et al.: The clinical development of percutaneous heart valve technology. J. Thorac. Cardiovasc. Surg. 129, 970 (2005)

4. Lutter, G., Ardehali, R., Cremer, J., et al.: Percutaneous valve replacement: current state and future prospects. Ann. Thor. Surg. 78, 2199-2206 (2004)

5. Modi, P., Rodriguez, E., Chitwood, W.R.J.: Robot-assisted cardiac surgery. Interact. Cardiovasc. Thorac. Surg. 9, 500-505 (2009)

6. Trejos, A.L., et al.: Optimizing port placement for robot-assisted minimally invasive cardiac surgery. Int. J. Med. Robotics Comput. Assist. Surg. 3, 355-364 (2007)

7. Damiano, R.Jr.: Robotics in cardiac surgery: The emperor's new clothes. The Journal of Thoracic and Cardiovascular Surgery 134(3), 559-561 (2007)

8. Moore, J.T., Wiles, A.D., et al.: Integration of trans-esophageal echocardiography with magnetic tracking technology for cardiac interventions. In: Medical Imaging 2010: Visualization, Image-Guided Procedures and Modeling. Proc. of SPIE, vol. 7625, pp. 76252Y-1-10 (2010)

9. Linte, C.A., Carias, M., et al.: Estimating heart shift and morphological changes during minimally invasive cardiac interventions. In: Medical Imaging 2010: Visualization, Image-Guided Procedures and Modeling. Proc. of SPIE, vol. 7625, pp. 762509-1-11 (2010)

10. Press, W.H., Flannery, B.P., et al.: Numerical recipies in C: The arts of scientific computing. Cambridge University Press, Cambridge (1992)

11. Ma, B., Ellis, R.E., Fleet, D.J.: Spotlights: A robust method for surface-based registration in orthopedic surgery. In: Taylor, C., Colchester, A. (eds.) MICCAI 1999. LNCS, vol. 1679, pp. 936-945. Springer, Heidelberg (1999) 